\title{
CASE REPORT: CONTINUOUS BILATERAL QUADRATUS LUMBORUM BLOCKS IN AN INFANT
}

\author{
Christian Bergek, MD, EDRA \\ Department of Paediatric Anaesthesia and Intensive Care \\ The Queen Silvia Children's Hospital, Sahlgrenska University Hospital, Gothenburg, Sweden. \\ cbergek@gmail.com
}

\section{Background}

Quadratus lumborum block (QLB) is becoming popular in paediatric anaesthesia but few mentions of continuous

Our patient was born at week 36 , with bladder exstrophy. He had surgery first at birth, and 2 months later for duodenal stenosis. Now, at 6 months, weighing $6.5 \mathrm{~kg}$ he had surgery again to close the bladder and the abdominal wall, and bilateral pelvic osteotomies to make it possible. Postoperatively he was confined to the supine position with the legs elevated in a stretching device to release tension in the surgical area.

Because of vertebral malformations (upper thorax and lower part of sacrum) any central block was deemed was given. Postoperatively he was kept intubated in PICU until stable analgesia and respiration, which turned out to be difficult to achieve. (Table 1)

\section{Methods}

At day 2 I performed bilateral QLB blocks with catheters. The catheters were secured with Dermabond ${ }^{\circledR}$ mini, and Tegaderm ${ }^{\mathrm{TM}}$. For practical reasons I did a transmuscular QLB on the right side and a QLB type 2 on the left side. (Fig 1\&2) Because of surgical dressings both injections were done from medial aiming laterally. Starting doses were $2.5 \mathrm{ml}$ of levobupivacaine $(1.6 \mathrm{mg} / \mathrm{ml})$ on each side. Thereafter $3 \mathrm{ml}$ levobupivacaine $(0.625 \mathrm{mg} / \mathrm{ml})$ on each side every 4 th hour. QLB exist. ${ }^{1,2}$ contraindicated. Peroperatively large doses of fentanyl

\section{Results}

The patient became calmer and other analgesics could be withdrawn step by step. Catheters were withdrawn after 9 days. No side effects were noted. (Table 1)

\section{Conclusions}

Continuous QLB seems applicable also in infants.
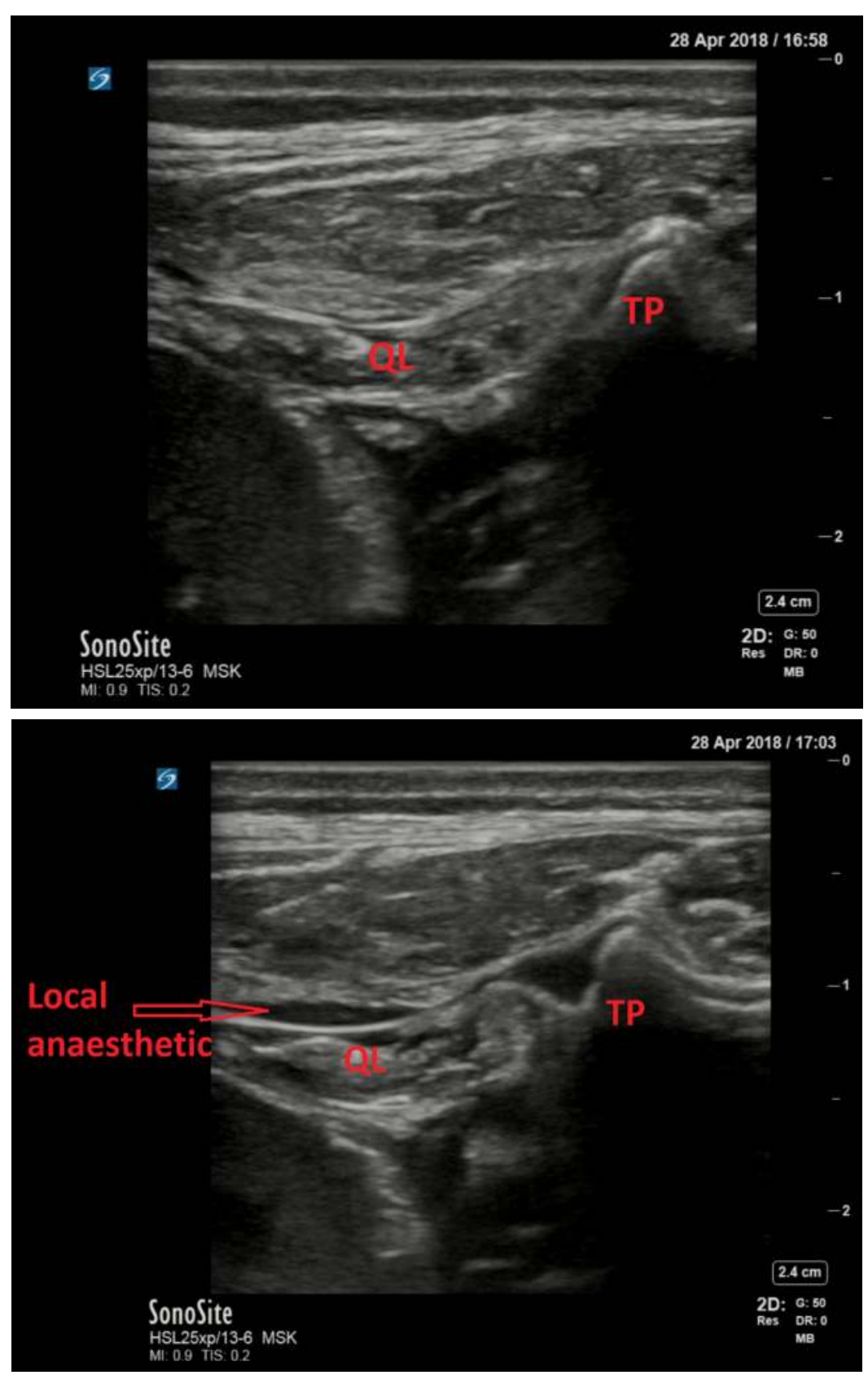

Fig 1\&2: QLB block type 2 on left side. Before and after injection.

\begin{tabular}{|c|c|c|c|c|c|c|c|c|c|c|c|c|c|}
\hline & $\begin{array}{l}\text { Day } 0= \\
\text { surgery }\end{array}$ & Day 1 & Day 2 & Day 3 & Day 4 & Day 5 & Day 6 & Day 7 & Day 8 & Day 9 & Day 10 & Day 11 & Day 12 \\
\hline $\begin{array}{l}\text { Opioids } \\
\mu \mathrm{g} / \mathrm{kg} / \mathrm{h}\end{array}$ & $\begin{array}{c}30 \\
\text { (morphine) }\end{array}$ & $35-40$ & $40-35$ & 35 & 30 & 20 & 15 & $\begin{array}{l}10 \\
\text { (ketobe- } \\
\text { midone) }\end{array}$ & $\rightarrow$ & $\rightarrow$ & $\rightarrow$ & $\rightarrow$ & \\
\hline Paracetamol & $\begin{array}{c}7.5 \mathrm{mg} / \mathrm{kg} \\
\text { every } 6^{\text {th }} \text { hour }\end{array}$ & $\rightarrow$ & $\rightarrow$ & $\rightarrow$ & $\rightarrow$ & $\rightarrow$ & $\rightarrow$ & $\rightarrow$ & $\rightarrow$ & $\rightarrow$ & $\rightarrow$ & $\rightarrow$ & $\rightarrow$ \\
\hline$\alpha 2$-agonists & $\begin{array}{l}\text { Dexmedeto- } \\
\text { midine } 1.4 \\
\mu \mathrm{g} / \mathrm{kg} / \mathrm{h}\end{array}$ & $\rightarrow$ & $\begin{array}{c}\text { Clonidine } \\
2.0 \mu \mathrm{g} / \mathrm{kg} / \mathrm{h}\end{array}$ & $\rightarrow$ & $\rightarrow$ & $\rightarrow$ & $\rightarrow$ & $\rightarrow$ & $\begin{array}{l}\text { Clonidine } \\
15 \mu \mathrm{g} 6 \\
\text { times daily }\end{array}$ & $\rightarrow$ & $\rightarrow$ & $\rightarrow$ & $\rightarrow$ \\
\hline $\begin{array}{l}\text { Ketamine } \\
\mathrm{mg} / \mathrm{kg} / \mathrm{h}\end{array}$ & & 0.2 & $\rightarrow$ & $\rightarrow$ & $\rightarrow$ & 0.1 & $\rightarrow$ & $\rightarrow$ & 0 & & & & \\
\hline NSAID & & & Ketorolac iv & $\rightarrow$ & $\rightarrow$ & $\rightarrow$ & $\rightarrow$ & $\rightarrow$ & $\begin{array}{l}\text { Ibuprofen } \\
\text { oral }\end{array}$ & $\rightarrow$ & $\rightarrow$ & $\rightarrow$ & $\rightarrow$ \\
\hline $\begin{array}{l}\text { Midazolam } \\
\mathrm{mg} / \mathrm{kg} / \mathrm{h}\end{array}$ & & 0.1 & 0.2 & 0.15 & 0.1 & 0 & & & & & & & \\
\hline Nerve block & & & QLB & $\begin{array}{c}3 \mathrm{ml} \\
\text { every } 4^{\text {th }} \\
\text { hour, } \\
\text { bilateral }\end{array}$ & & & & & & $\begin{array}{c}3 \mathrm{ml} \\
\text { every } 6^{\text {th }} \\
\text { hour, } \\
\text { bilateral }\end{array}$ & $\begin{array}{c}3 \mathrm{ml} \\
\text { every } 8^{\text {th }} \\
\text { hour, } \\
\text { bilateral }\end{array}$ & $\begin{array}{c}3 \mathrm{ml} \\
\text { every } \\
12^{\text {th }} \text { hour, } \\
\text { bilateral }\end{array}$ & $\begin{array}{c}3 \mathrm{ml} \text { once } \\
\text { bilateral }\end{array}$ \\
\hline $\begin{array}{l}\text { Propofol } \\
\mathrm{mg} / \mathrm{kg} / \mathrm{h}\end{array}$ & 2 & $\rightarrow$ & $\rightarrow$ & $\rightarrow$ & $\rightarrow$ & 0 & & & & & & & \\
\hline Comments & \multicolumn{3}{|c|}{$\begin{array}{l}\text { Pain, ventilator asynchrony, muscle } \\
\text { relaxation, boluses }\end{array}$} & $\begin{array}{l}\text { More at } \\
\text { ease. } \\
\text { Less } \\
\text { boluses }\end{array}$ & $\begin{array}{l}\text { Can be } \\
\text { handled } \\
\text { without } \\
\text { protests, } \\
\text { wakes up } \\
\text { calmly }\end{array}$ & $\begin{array}{l}\text { Extub- } \\
\text { ation }\end{array}$ & & & $\begin{array}{l}\text { Leaves } \\
\text { PICU for } \\
\text { paediatric } \\
\text { surgical } \\
\text { ward }\end{array}$ & & & & $\begin{array}{l}\text { Catheters } \\
\text { extracted }\end{array}$ \\
\hline
\end{tabular}

